\title{
Conocimientos y actitudes en alimentación y actividad física de los residentes de medicina familiar de una universidad en Bogotá, Colombia
}

\section{Attitude and knowledge in healthy eating and physical activity of family medicine residents from university in Bogotá, Colombia}

\author{
Gustavo Alfonso Díaz Muñoz ${ }^{1 *}$, Natalia Buitrago Sierra², Sión Daniela Gómez Avendaño², \\ Mariana Gutiérrez Mojica², Carolina Ramírez Pallares²
}

Recibido: 5 de febrero de 2019. Aceptado para publicación: 12 de marzo de 2019

Publicado en línea, marzo 23 de 2019

https://doi.org/10.35454/rncm.v2n1.061

\section{Resumen}

Introducción: la modificación de factores de riesgo mediante promoción de estilos de vida saludable es una estrategia costoefectiva en el control de las enfermedades no transmisibles. Al médico familiar corresponde promocionarlas, para lo cual requiere adecuados conocimientos y actitudes que le permitan una comunicación asertiva con sus pacientes.

Objetivo: identificar los conocimientos y actitudes en nutrición, y actividad física de los residentes de medicina familiar de la Universidad El Bosque en Bogotá.

Metodología: estudio transversal descriptivo. Se diseñó una encuesta de conocimientos y actitudes sobre alimentación saludable y actividad física, que se aplicó a residentes del programa de medicina familiar en agosto de 2017. Se reportaron frecuencias, porcentajes, promedios y desviaciones estándar. Se empleó la prueba de Ji2 para las comparaciones entre sexo y años de residencia, asumiendo un valor $\mathrm{p}<0,05$.

Resultados: participaron 55 estudiantes ( $77 \%$ de la población), con edad media de 31,6 años (+/-3,44) y 65,5\% fueron mujeres. El porcentaje de respuestas correctas en nutrición y actividad física fueron $73 \%$ y $77 \%$ respectivamente, sin diferencia entre años de residencia $(p>0,05)$. El 72,7 \% consideran que la consejería/valoración nutricional deben incluirse en la consulta y solo 38,2 \% y 40 $\%$ de los participantes se sienten cómodos abordando temas de nutrición y actividad física respectivamente.

Conclusiones: se encontraron actitudes favorables hacia la nutrición y la actividad física; los vacíos de conocimiento en nutrición y actividad física son relevantes y requieren de una intervención en el currículo del programa.

Palabras clave: dieta saludable, ejercicio, conocimientos, actitudes y práctica en salud, medicina familiar y comunitaria.

1 Instituto de Investigación en Nutrición, Genética y Metabolismo, Facultad de Medicina, Universidad El Bosque. Bogotá, D.C., Colombia.

\section{Summary}

Background: The modification of risk factors through the promotion of healthy lifestyles is a cost-effective strategy in the control of noncommunicable diseases. The family doctor is responsible for promoting them, for which it requires adequate knowledge and attitudes that allow an assertive communication with their patients.

Objective: Identify the knowledge and attitudes in nutrition and physical activity of the family medicine residents of El Bosque University in Bogotá.

Methods: Descriptive cross-sectional study. A survey of knowledge and attitudes about healthy eating and physical activity was designed, which was applied to residents of the family medicine program in August-2017. Frequencies, percentages, averages and standard deviations were reported. The Chi2 test was used for comparisons between sex and years of residence, assuming a value $\mathrm{p}<0.05$.

Results: The research involved 55 students ( $77 \%$ of the population), with an average age of 31.6 years (+/- 3.44) and $65.5 \%$ were women. The percentage of correct answers in nutrition and physical activity were $73 \%$ and $77 \%$ respectively, with no difference between years of residence ( $p>0.05$ ). $72.7 \%$ consider that nutritional counseling / assessment should be included in the consultation and only $38.2 \%$ and $40 \%$ of participants feel comfortable addressing nutrition and physical activity issues respectively.

Conclusions: Favorable attitudes towards nutrition and physical activity were found; knowledge gaps in nutrition and physical activity are relevant and require prompt attention from the program.

Keywords: Healthy Diet; Exercise; Knowledge; Attitudes; Practice; Family Practice.

2 Facultad de Medicina, Universidad El Bosque. Bogotá, D.C., Colombia.

*Correspondencia: Gustavo Alfonso Díaz Muñoz

diazgustavo@unbosque.edu.co 


\section{INTRODUCCIÓN}

Las enfermedades no transmisibles (ENT) son la primera causa de morbi-mortalidad y de discapacidad en el mundo. Representan una carga social y económica para los países y su prevención está incluida en los objetivos de desarrollo sostenible número 3: "Garantizar una vida sana y promover el bienestar para todos en todas las edades"(1). La promoción de estilos de vida saludable es una estrategia costo-efectiva en el control de estas enfermedades ${ }^{(2)}$ y el médico familiar se encuentra en primera línea para aconsejar a los pacientes en la adopción de estas conductas. Además, los pacientes consideran a los médicos como una fuente y ejemplo fiable en temas de nutrición y actividad física ${ }^{(3-5)}$.

El médico familiar es el encargado de realizar la prevención primaria y secundaria en las diferentes etapas del ciclo vital y tiene un papel en la implementación de las estrategias de cambio de hábitos de vida saludables. Para lo anterior, es necesario tener conocimientos y competencias necesarias para abordar estos temas. A pesar de lo anterior, algunos estudios han señalado que la educación en nutrición y actividad física es escasa en las facultades del área de la salud en pregrado y posgrado $^{(6-11)}$, lo que podría afectar negativamente la identificación oportuna de alteraciones nutricionales y la asesoría en temas de alimentación y actividad física ${ }^{(12)}$.

Determinar los conocimientos y actitudes en nutrición y actividad física en estudiantes de medicina familiar, permitiría conocer qué tan motivados y conscientes acerca de la importancia del tema están los residentes y posibilitaría la identificación de oportunidades de mejora en los currículos. Por lo anterior, este estudio tiene como objetivo describir los conocimientos y actitudes en alimentación saludable y actividad física de los residentes de Medicina Familiar de la Universidad El Bosque en Bogotá, Colombia.

\section{MATERIALES Y MÉTODOS}

\section{Diseño del estudio}

Se realizó un estudio observacional y descriptivo en estudiantes de los tres años del posgrado de medicina familiar de la Universidad El Bosque durante el mes de agosto de 2017.

\section{Población}

El universo del estudio fueron los 73 estudiantes del posgrado de Medicina Familiar de la Universidad El Bosque durante agosto de 2017. El tamaño de muestra no se calculó y en cambio se convocó a todos los residentes de primero, segundo y tercer año de residencia del posgrado. El muestreo fue por intención de participar. Se incluyeron estudiantes matriculados en el posgrado, que aceptaron participar en el estudio y realizaron el proceso de consentimiento informado. No se aplicaron criterios de exclusión.

\section{Mediciones}

Se recolectó información demográfica (sexo y edad) y académica (año de residencia, antecedentes de educación en nutrición y actividad física). La evaluación de actitud frente a la nutrición y la actividad física se midieron mediante 12 afirmaciones a las que se contestó en una escala Likert de cinco opciones (total desacuerdo, desacuerdo, ni acuerdo ni en desacuerdo, acuerdo y muy de acuerdo) y asumiendo el "acuerdo" y "muy de acuerdo" como actitud favorable hacia cada una de las afirmaciones. Las preguntas de actitudes se basaron en la encuesta realizada en Colombia Healthy Doctor $=$ Healthy Patient ${ }^{(13)} \mathrm{y}$ en las publicaciones de McGaghie et al ${ }^{(14)}$, Mihalynuk et al ${ }^{(15)}$ y Vetter M et $a l^{(6)}$.

Para evaluar los conocimientos en alimentación saludable y actividad física se elaboró un cuestionario de 28 preguntas de selección múltiple con una única respuesta, basado en las Guías Alimentarias Basadas en Alimentos para la población colombiana mayor de 2 años ${ }^{(16)}$ y en las recomendaciones mundiales sobre actividad física para la salud ${ }^{(17)}$. Para la recolección de información se utilizó el aplicativo de google formularios, el cual se envió vía correo electrónico y se diligenció durante una clase presencial donde estaban presentes todos los residentes.

\section{Análisis estadístico}

Las variables categóricas se describieron a través de frecuencias y porcentajes y las variables cuantitativas mediante promedios, desviaciones estándar (+/-), medianas y valores extremos. Para el análisis bivariado la variable de agrupación fue el sexo y el año de residencia, utilizando las pruebas estadísticas de $\mathrm{Chi}^{2}$ y U-MannWhitney; para todas las pruebas se asumió como significativo un valor $\mathrm{p}<0,05$. Se empleó el software estadístico SPSS V.21 licenciado para la Universidad El Bosque.

\section{Declaración ética}

Esta investigación cumplió los principios éticos de la Declaración de Helsinki, de la Asociación Médica 
Mundial, de la Resolución 8430 de 1993 del Ministerio de Salud de Colombia, contó con la revisión y aprobación del Comité de Ética ad hoc de la Universidad El Bosque (UB 448-2017 junio 23 de 2017), la participación de los estudiantes fue voluntaria y se realizó el proceso de consentimiento informado por escrito.

\section{RESULTADOS}

Participaron $55(75,3 \%)$ de los 73 estudiantes de Medicina Familiar, con una edad media de 31,6 años $(+/-3,4)$ y $65,5 \%$ fueron mujeres. Solo $58,2 \%$ de los participantes han tenido educación o formación extracurricular en nutrición o actividad física (congresos, diplomados, cursos o talleres), cerca de $30 \%$ recibió cursos de actividad física y nutrición durante la carrera de medicina y no se evidenciaron diferencias por sexo $(\mathrm{p}>0,05)$ (Tabla 1$)$.

Respecto a la actitud favorable hacia la nutrición, 38,2 \% "se sienten cómodos abordando el tema de la nutrición con los pacientes" y el 100 \% manifestó estar de acuerdo en que "todos los médicos, independientemente de la especialidad, deben aconsejar a sus pacientes con riesgo sobre cambios en la dieta" (Tabla 2). En acti-

Tabla 1. Descripción de la población

\begin{tabular}{|c|c|c|c|c|}
\hline & $\begin{array}{c}\text { Mujeres } \\
\text { n } 36(65,5 \%)\end{array}$ & $\begin{array}{c}\text { Hombres } \\
\text { n } 19(34,5 \%)\end{array}$ & $\begin{array}{l}\text { Total } \\
\text { n } 55\end{array}$ & Valor $p$ \\
\hline Edad: años * & $\begin{array}{l}31,4(3,17) \\
32(25-40)\end{array}$ & $\begin{array}{c}31,8(3,9) \\
32(25-40)\end{array}$ & $\begin{array}{l}31,6(3,44) \\
32(25-40)\end{array}$ & 0,792 \\
\hline $\begin{array}{c}\text { Año de Residencia: ** } \\
1 \\
2 \\
3\end{array}$ & $\begin{array}{l}13(36,1) \\
13(36,1) \\
10(27,8)\end{array}$ & $\begin{array}{l}11(57,9) \\
4(21,1) \\
4(21,1)\end{array}$ & $\begin{array}{l}24(43,6) \\
17(30,9) \\
14(25,5)\end{array}$ & 0,289 \\
\hline Con educación continuada $* *$ & $19(52,8)$ & $13(68,4)$ & $32(58,2)$ & 0,263 \\
\hline Con curso de nutrición durante la carrera $* *$ & $12(33,3)$ & $5(26,3)$ & $17(30,9)$ & 0,592 \\
\hline Con curso de actividad física durante la carrera ** & $11(30,6)$ & $3(15,8)$ & $14(25,5)$ & 0,334 \\
\hline Familiar con apoyo nutricional ** & $20(55,6)$ & $8(42,1)$ & $28(50,9)$ & 0,343 \\
\hline
\end{tabular}

* Valores presentados como promedios y (desviación estándar)

** Valores presentados como frecuencias y (\%)

Tabla 2. Actitud favorable hacia la nutrición por año de residencia

\begin{tabular}{|c|c|c|c|c|}
\hline & $\begin{array}{c}\text { Año } 1 \text { de } \\
\text { residencia } \\
\text { n } 24(43,6 \%)\end{array}$ & $\begin{array}{l}\text { Año } 2 \text { de } \\
\text { residencia } \\
\text { n } 17(30,9 \%)\end{array}$ & $\begin{array}{c}\text { Año } 3 \text { de } \\
\text { residencia } \\
\text { n } 14(25,4 \%)\end{array}$ & $\begin{array}{l}\text { Total } \\
\text { n } 55\end{array}$ \\
\hline $\begin{array}{l}\text { Todos los médicos, independientemente de la especialidad, deben } \\
\text { aconsejar a sus pacientes con riesgo, sobre cambios en la dieta. }{ }^{*}\end{array}$ & $24(100)$ & $17(100)$ & $14(100)$ & $55(100)$ \\
\hline La consejería y valoración nutricional debe incluirse en toda consulta. * & $18(75)$ & $13(76,5)$ & $9(64,3)$ & $40(72,7)$ \\
\hline $\begin{array}{l}\text { Tengo la obligación de mejorar la salud de mis pacientes incluyendo } \\
\text { la discusión sobre la nutrición con ellos. }{ }^{*}\end{array}$ & $18(75)$ & $15(88,2)$ & $12(85,7)$ & $45(81,8)$ \\
\hline $\begin{array}{l}\text { El médico puede tener un efecto sobre el comportamiento } \\
\text { alimentario del paciente si tiene el tiempo durante la consulta para } \\
\text { tratar el problema. }{ }^{*}\end{array}$ & $21(87,5)$ & $14(82,4)$ & $12(85,7)$ & $47(85,5)$ \\
\hline $\begin{array}{l}\text { La consejería nutricional no es de mi competencia y por tanto no } \\
\text { debo abordar ese tema en la consulta con mis pacientes. Remitiré los } \\
\text { pacientes a la nutricionista. }{ }^{*}\end{array}$ & $18(75)$ & $11(64,7)$ & $8(57,1)$ & $37(67,3)$ \\
\hline $\begin{array}{l}\text { Me siento cómodo abordando el tema de la nutrición con mis } \\
\text { pacientes. * }\end{array}$ & $7(29,2)$ & $9(52,9)$ & $5(35,7)$ & $21(38,2)$ \\
\hline
\end{tabular}

* Valores reportados como frecuencia y (\%) 
vidad física, 94,5 \% tiene actitud favorable hacia "recomendar cambios para lograr estilos de vida saludable es importante en cualquier etapa de ciclo vital" y $40 \%$ se siente cómodo abordando el tema de la actividad física con los pacientes (Tabla 3).

Con relación a los conocimientos, los residentes obtuvieron $77 \%$ y $73 \%$ de respuestas acertadas en actividad física y nutrición respectivamente, el conocimiento en actividad física es similar entre los tres años de residencia y el conocimiento en nutrición solo es superior en el tercer año de residencia (Figura 1).

Se encontró un alto porcentaje de residentes con respuestas acertadas (>90\%) en las preguntas de cálculo e interpretación del IMC, importancia de la alimentación saludable, alimentos para la salud de músculos-huesosdientes, omitir comida chatarra para mantener un peso saludable, consumo de sodio y tensión arterial, gluten y enfermedad celiaca, lactancia materna exclusiva y suplementación de ácido fólico/hierro en el embarazo. El tema "recomendación de actividad física en adultos" tuvo un alto porcentaje de respuesta acertada (>90\%) (Tabla 4).

\section{DISCUSIÓN}

Este es el primer reporte en Colombia y Latinoamérica que describe en residentes de Medicina Familiar sus conocimientos y actitudes hacia la nutrición y actividad física. Además, se evidencia la persistencia o inmu- tabilidad de dichos conocimientos y actitudes durante la formación de los futuros médicos especialistas en Medicina Familiar.

\section{Actitudes}

El estudio mostró que los residentes expresan una actitud favorable frente al abordaje de hábitos alimentarios y actividad física. Si bien la actitud es favorable y la mayoría de los participantes $(72,7 \%)$ considera que es de su competencia implementar la consejería en dichos temas durante la consulta, $38 \%$ de los participantes no se siente cómodo al hablar de actividad física y $40 \%$ sobre nutrición en la consulta. Lo anterior es semejante a lo reportado en el estudio de Vetter et al. en Estados Unidos ${ }^{(6)}$. Este estudio evaluó a 114 residentes de medicina interna y encontró que $77 \%$ estaba de acuerdo con incluir la evaluación nutricional en las visitas rutinarias de atención primaria, 94 \% refirió que era obligación discutir de nutrición con los pacientes, $11 \%$ no se siente cómodo hablando de actividad física y entre $42 \%$ - $65 \%$ no se sintió cómodo hablando sobre diversos temas de nutrición.

Para la realización de consejería eficaz en alimentación y actividad física es necesario contar con el conocimiento y con el interés suficientes del médico para realizar estas intervenciones dentro de la consulta. Todos los participantes del estudios consideran que el

Tabla 3. Actitud favorable hacia la actividad física por año de residencia

\begin{tabular}{|c|c|c|c|c|}
\hline & $\begin{array}{c}\text { Año } 1 \text { de } \\
\text { residencia } \\
\text { n } 24(43,6 \%)\end{array}$ & $\begin{array}{c}\text { Año } 2 \text { de } \\
\text { residencia } \\
\text { n } 17(30,9 \%)\end{array}$ & $\begin{array}{c}\text { Año } 3 \text { de } \\
\text { residencia } \\
\text { n } 14(25,4 \%)\end{array}$ & $\begin{array}{l}\text { Total } \\
\text { n } 55\end{array}$ \\
\hline $\begin{array}{l}\text { Recomendar cambios para lograr estilos de vida saludable es } \\
\text { importante en cualquier etapa de ciclo vital. * }\end{array}$ & $22(91,7)$ & 17 (100) & $13(92,9)$ & $52(94,5)$ \\
\hline $\begin{array}{l}\text { Los médicos tienen la responsabilidad de lograr que los } \\
\text { individuos adopten estilos de vida saludables. }{ }^{*}\end{array}$ & $15(62,5)$ & $9(52,9)$ & $8(57,1)$ & $32(58,2)$ \\
\hline $\begin{array}{l}\text { La consejería sobre actividad física debe incluirse en toda } \\
\text { consulta. * }\end{array}$ & $18(75)$ & $8(47,1)$ & $8(57,1)$ & $34(61,8)$ \\
\hline $\begin{array}{l}\text { El médico puede tener un efecto sobre la actividad física del } \\
\text { paciente si tiene el tiempo durante la consulta para tratar el } \\
\text { problema. }{ }^{*}\end{array}$ & $16(66,7)$ & $11(64,7)$ & $10(71,4)$ & $37(67,3)$ \\
\hline $\begin{array}{l}\text { La consejería sobre actividad física no es de mi competencia y por } \\
\text { tanto no debo abordarlo en la consulta con mis pacientes. }{ }^{*}\end{array}$ & $18(75)$ & $16(94,1)$ & $9(64,3)$ & $43(78,2)$ \\
\hline $\begin{array}{l}\text { Me siento cómodo abordando el tema de la actividad física con } \\
\text { mis pacientes. * }\end{array}$ & $8(33,3)$ & $8(47,1)$ & $6(42,9)$ & $22(40)$ \\
\hline
\end{tabular}

* Valores reportados como frecuencia y (\%) 


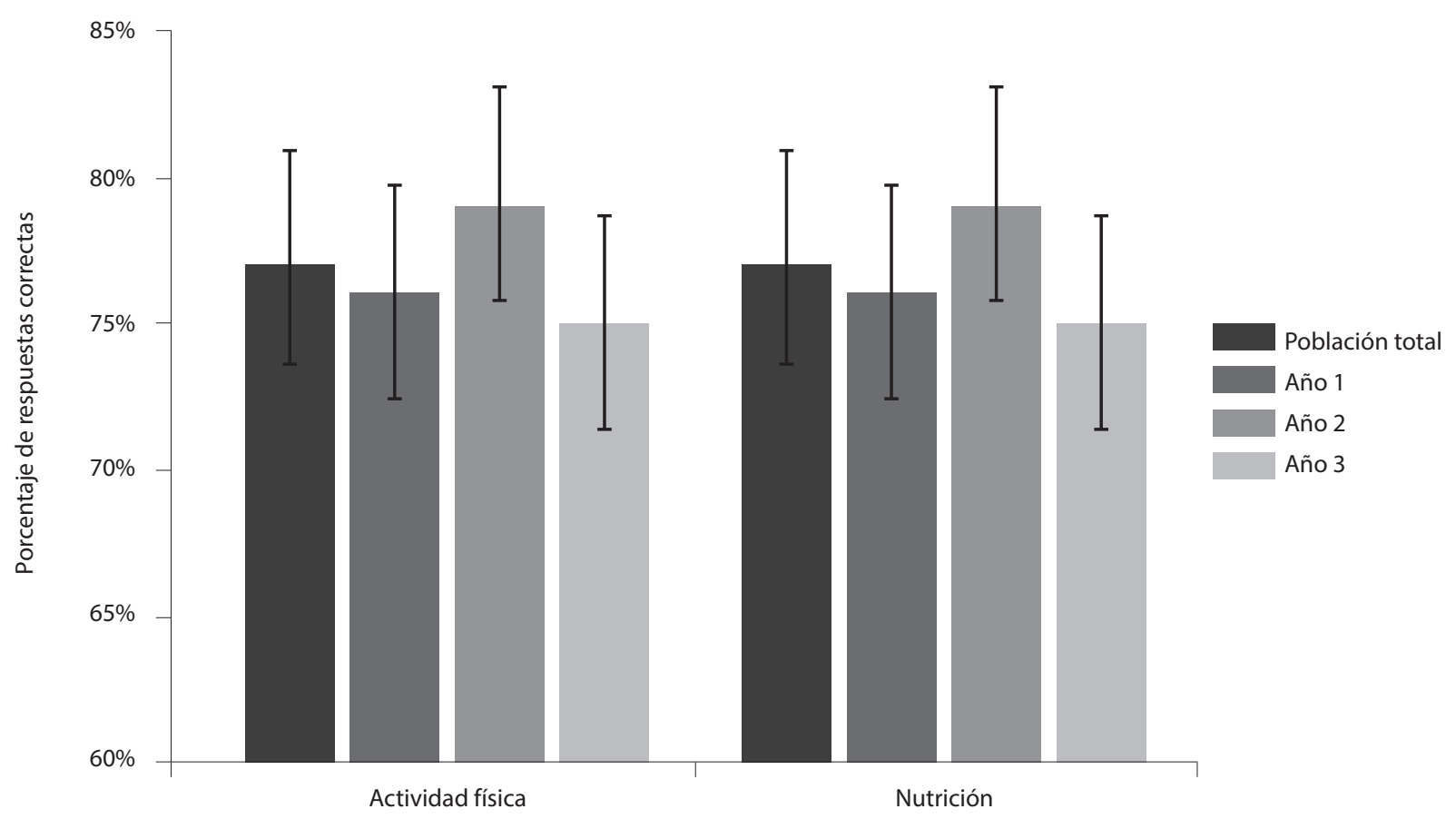

Figura 1. Porcentaje de respuestas correctas en conocimientos de nutrición y actividad física, por año de residencia.

médico debe recomendar cambios nutricionales para prevenir enfermedades crónicas no transmisibles, lo cual coincide con lo reportado en el estudio de Crowley et al. en Nueva Zelanda ${ }^{(18)}$ y Nasser et al. en Canadá( ${ }^{(19)}$.

\section{Conocimientos}

Este estudio evidencia que los residentes de medicina familiar reconocen la importancia de aconsejar sobre alimentación saludable y actividad física, pero de acuerdo con Gautreau et al. ${ }^{(20)}$ y Etherton et al. ${ }^{(21,22)}$ la falta de conocimientos puede ser considerada una barrera para promoverlos.

La ausencia de preparación de los médicos para proporcionar a los pacientes atención nutricional ha sido descrita desde hace varias décadas ${ }^{(20,23)}$. Estudios que han investigado la situación de la educación nutricional en el plan de estudios de escuelas de medicina, han mostrado que tanto los médicos en ejercicio como los estudiantes de medicina aseguran que han recibido una educación nutricional inadecuada ${ }^{(15,18,19,24-27)}$. Sin embargo, pocos estudios han evaluado los conocimientos en nutrición de estudiantes de Medicina Familiar. En Estados Unidos, el estudio de Oberhelman et al. ${ }^{(28)}$ en 13 residentes de medicina familiar describió conocimientos relacionados con la suplementación de vitamina D.

La encuesta permite mostrar vacíos conceptuales en nutrición y actividad física (26\% de respuestas incorrectas), como el plato saludable o los beneficios de la actividad física para la salud, lo que concuerdan con el estudio de Vetter et al. ${ }^{(6)}$, donde los residentes obtuvieron un promedio de $34 \%$ de respuestas incorrectas.

No se indagó sobre las causas o factores relacionados con los conocimientos en nutrición y actividad física, pero los resultados podrían deberse a uno o más factores que Mogre et al. ${ }^{(29)}$ describen en 23 estudiantes de medicina de quinto año: falta de prioridad de este tema, falta de profesores para proporcionar educación nutricional, deficiente aplicación de la ciencia de la nutrición a la práctica clínica y la pobre colaboración con profesionales de la nutrición durante la formación.

Hay que resaltar que no hay estudios publicados sobre las actitudes y los conocimientos en alimentación saludable y actividad física de residentes de alguna especialidad médica en Colombia. Solo el estudio de Duperly et al. ${ }^{(13)}$ en el marco del programa "Médico saludable = paciente saludable", muestra la asociación positiva entre las conductas saludables y las actitu- 
Tabla 4. Estudiantes con calificación acertada en cada pregunta de conocimiento en nutrición y actividad física

\begin{tabular}{|c|c|c|c|c|c|}
\hline & & $\begin{array}{c}\text { Año } 1 \text { de } \\
\text { residencia } \\
\text { n } 24 \\
\%\end{array}$ & $\begin{array}{c}\text { Año } 2 \text { de } \\
\text { residencia } \\
\text { n } 17 \\
\%\end{array}$ & $\begin{array}{c}\text { Año } 3 \text { de } \\
\text { residencia } \\
\text { n } 14 \\
\%\end{array}$ & $\begin{array}{c}\text { Total } \\
\text { n } 55 \\
\%\end{array}$ \\
\hline \multicolumn{2}{|c|}{ Nutrición * } & & & & \\
\hline 1 & Cálculo de IMC & $22(91,7)$ & $16(94,1)$ & $12(85,7)$ & $50(90,9)$ \\
\hline 2 & IMC para diagnóstico obesidad & $24(100)$ & $16(94,1)$ & $14(100)$ & $54(98,2)$ \\
\hline 3 & Conoce el plato saludable de Colombia & $6(25)$ & $7(41,2)$ & $8(57,1)$ & $21(38,2)$ \\
\hline 4 & Lo importante de una alimentación saludable & $20(83,3)$ & $16(94,1)$ & $14(100)$ & $50(90,9)$ \\
\hline 5 & Grupo de alimentos de cereales & $1(4,2)$ & $2(11,8)$ & 0 & $3(5,5)$ \\
\hline 6 & Alimentos para la salud de músculos, huesos y dientes & $23(95,8)$ & $13(76,5)$ & $14(100)$ & $50(90,9)$ \\
\hline 7 & Consumo de Frutas y verduras para la digestión y salud cardiovascular & $21(87,5)$ & $14(82,4)$ & $14(100)$ & $49(89,1)$ \\
\hline 8 & Consumo de frutos secos y aguacate para mejorar el colesterol & $17(70,8)$ & $10(58,8)$ & $13(92,9)$ & $40(72,7)$ \\
\hline 9 & Omitir comida chatarra para mantener un peso saludable & $22(91,7)$ & $16(94,1)$ & $13(92,9)$ & $51(92,7)$ \\
\hline 10 & Recomendación de consumo de leguminosas & $19(79,2)$ & $16(94,1)$ & $13(92,9)$ & $48(87,3)$ \\
\hline 11 & Reducción del consumo de sodio para tensión arterial normal & $23(95,8)$ & $16(94,1)$ & $13(92,9)$ & $52(94,5)$ \\
\hline 12 & Grasas saturadas asociadas al aumento de colesterol LDL & $22(91,7)$ & $14(82,4)$ & $11(78,6)$ & $47(85,5)$ \\
\hline 13 & Consumo de gluten y enfermedad celiaca & $22(91,7)$ & $14(82,4)$ & $14(100)$ & $50(90,9)$ \\
\hline 14 & Requerimiento diario de Vitamina $D$ en menores de 1 año & $14(58,3)$ & $6(35,3)$ & $10(71,4)$ & $30(54,5)$ \\
\hline 15 & Alimentos para la prevención de anemia & $10(41,7)$ & $8(47,1)$ & $5(35,7)$ & $23(41,8)$ \\
\hline 16 & Recomendaciones para disminuir el riesgo cardiovascular & $4(16,7)$ & $11(64,7)$ & $6(42,9)$ & $21(38,2)$ \\
\hline 17 & Consumo de calcio en la infancia & $9(37,5)$ & $2(11,8)$ & $5(35,7)$ & $16(29,1)$ \\
\hline 18 & Recomendación lactancia materna exclusiva & $24(100)$ & $16(94,1)$ & $14(100)$ & $54(98,2)$ \\
\hline 19 & introducción de leche de vaca en complementaria & $13(54,2)$ & $10(58,8)$ & $13(92,9)$ & $36(65,5)$ \\
\hline 20 & Suplementación de Ácido fólico y hierro en embarazo & $22(91,7)$ & $15(88,2)$ & $13(92,9)$ & $50(90,9)$ \\
\hline \multicolumn{2}{|c|}{ Actividad física * } & & & & \\
\hline 1 & La actividad física es una prescripción médica & $20(83,3)$ & $15(88,2)$ & $13(92,9)$ & $48(87,3)$ \\
\hline 2 & Actividad física para la prevención y manejo de enfermedades crónicas & $13(54,2)$ & $6(35,3)$ & $4(28,6)$ & $23(41,8)$ \\
\hline 3 & Definición de actividad física & $12(50)$ & $5(29,4)$ & $6(42,9)$ & $23(41,8)$ \\
\hline 4 & Recomendación de actividad física en adultos & $24(100)$ & $17(100)$ & $14(100)$ & $55(100)$ \\
\hline 5 & $\begin{array}{l}\text { Estilos de vida saludable del médico favorecen la adopción de los mismos } \\
\text { en el paciente }\end{array}$ & $24(100)$ & $16(94,1)$ & $14(100)$ & $54(98,2)$ \\
\hline 6 & Recomendación de entrenamiento de fuerza muscular en el adulto & $20(83,3)$ & $17(100)$ & $12(85,7)$ & $49(89,1)$ \\
\hline 7 & Tiempo de actividad física moderada/intensa en $<18$ años & $17(70,8)$ & $13(76,5)$ & $13(92,9)$ & $43(78,2)$ \\
\hline 8 & Recomendación de actividad física en adultos mayores & $20(83,3)$ & $12(70,6)$ & $9(64,3)$ & $41(74,5)$ \\
\hline
\end{tabular}

* Valores reportados como frecuencia y (\%) 
des favorables hacia el asesoramiento preventivo, en estudiantes de pregrado de medicina, lo cual también sucede en otros países ${ }^{(30)}$.

Este estudio solo describe el grado de conocimientos y actitudes en nutrición y actividad física, pero el conocimiento no es suficiente para lograr cambios en los pacientes, también es necesario superar otras barreras. Por lo tanto, otros temas de investigación en el futuro serían: a. identificar estrategias y su efecto para el mejoramiento de actitudes, prácticas y habilidades en nutrición y actividad física de estudiantes de medicina ${ }^{(31)}$; b. determinar o definir las habilidades en nutrición y actividad física para el médico especialista en Medicina Familiar ${ }^{(32)}$.

\section{Limitaciones del estudio}

A pesar de que esta investigación contó con la participación de residentes de los tres años de la especialidad y con un alto porcentaje de participación (75,3\% de la población total), el tamaño de muestra no fue suficiente para poder realizar todas las comparaciones del análisis bivariado, sugiriendo que se debe aumentar el número de participantes en futuras investigaciones, lo que podría lograrse incluyendo otras universidades con el programa de Medicina Familiar.

\section{CONCLUSIÓN}

Se encontró en la población actitudes favorables hacia la nutrición y la actividad física y se evidencian altos porcentaje de respuestas acertadas en los temas de actividad física. Sin embargo, los vacíos de conocimiento en nutrición requieren una pronta intervención desde el programa de Medicina Familiar.

\section{Declaración de autoría}

Todos los autores contribuyeron en forma significativa en el diseño del estudio, recolección y análisis de la información. De igual forma participaron en la redacción del artículo y aprobaron la versión final del mismo.

\section{Agradecimientos}

A Diana Cárdenas por dirigir el trabajo de grado de donde deriva este artículo.

\section{Conflicto de intereses}

Los autores declaran no tener conflicto de intereses.

\section{Financiación}

El presente estudio no tuvo financiación.

\section{Referencias bibliográficas}

1. World Health Organization. Objetivos de desarrollo sostenible [Internet]. Citado 01 junio, 2018. Disponible en: https:// www.un.org/sustainabledevelopment/es/news/communications-material/.

2. World Health Organization. Global action plan for the prevention and control of noncommunicable diseases 2013-2020. Switzerland: 2013. Citado 01 junio 2018. Disponible en: https://www.who.int/nmh/events/ncd_action_plan/en/

3. Kolasa KM, Rickett K. Barriers to providing nutrition counseling cited by physicians: A survey of primary care practitioners. Nutr Clin Pract. 2010;25(5):502-9.

4. Arciniegas Calle MC, Lobelo F, Jiménez MA, et al. One-day workshop-based training improves physical activity prescription knowledge in latin american physicians: A pre-test posttest study. BMC Public Health. 2016;16(1):1224.

5. Lobelo F, de Quevedo IG. The evidence in support of physicians and health care providers as physical activity role models. Am J Lifestyle Med. 2016;10(1):36-52.

6. Vetter ML, Herring SJ, Sood M, Shah NR, Kalet AL. What do resident physicians know about nutrition? an evaluation of attitudes, self-perceived proficiency and knowledge. J Am Coll Nutr. 2008;27(2):287-98.

7. Lo C. Integrating nutrition as a theme throughout the medical school curriculum. Am J Clin Nutr. 2000;72(Suppl 3):9S.

8. The report to congress on the appropriate federal role in assuring access by medical students, residents, and practicing physicians to adequate training in nutrition. Public Health Rep. 1994;109(6):824-6.

9. Adams KM, Kohlmeier M, Powell M, Zeisel SH. Nutrition in medicine: Nutrition education for medical students and residents. Nutr Clin Pract. 2010;25(5):471-80.

10. Freedhoff $Y$. The physician's role in cultivating healthful lifestyles. CMAJ. 2016;188(13):933-4.

11. Cardinal BJ, Park EA, Kim M, Cardinal MK. If exercise is medicine, where is exercise in medicine? review of U.S. medical education curricula for physical activity-related content. J Phys Act Health. 2015;12(9):1336.

12. Doubova SV, Pérez Cuevas R. Magnitud de las brechas en el diagnóstico y consejería nutricional para niños de uno a 5 años de edad en medicina familiar en méxico: Análisis de los datos del expediente electrónico. Atención Primaria. 2015;48(1):64-6.

13. Duperly J, Lobelo F, Segura C, Sarmiento F, Herrera D, Sarmiento OL, Frank E. The association between colombian medical students' healthy personal habits and a positive attitude toward preventive counseling: Cross-sectional analyses. BMC Public Health. 2009;9:218. 
14. McGaghie WC, Van Horn L, Fitzgibbon M, et al. Development of a measure of attitude toward nutrition in patient care. Am J Prev Med. 2001;20(1):15-20.

15. Mihalynuk TV, Coombs JB, Rosenfeld ME, Scott CS, Knopp RH. Survey correlations: Proficiency and adequacy of nutrition training of medical students. J Am Coll Nutr. 2008;27(1):59-64.

16. Instituto Colombiano de Bienestar Familiar. Guías alimentarias basadas en alimentos para la población colombiana mayor de 2 años. Bogotá: 2015. Citado 01 junio 2018. Disponible en: https://www.icbf.gov.co/bienestar/nutricion/educacionalimentaria

17. Organización Mundial de la Salud. Recomendaciones mundiales sobre la actividad física para la salud. Suiza: 2010. Citado 01 junio 2018. Disponible en: https://www.who.int/ dietphysicalactivity/publications/9789241599979/es/

18. Crowley J, Ball L, Han DY, Arroll B, Leveritt M, Wall C. New Zealand medical students have positive attitudes and moderate confidence in providing nutrition care: A cross-sectional survey. J Biomed Educ. 2015:259653. Citado 01 junio 2018. Disponible en: https://www.hindawi.com/journals/ jbe/2015/259653/

19. Nasser R, Heyland D, Wicklum S, et al. Medical students' perceptions of nutrition education in canadian universities. Appl Physiol Nutr Metab. 2010;35(3):336-43.

20. Gautreau S, Monsen ER. Priorities of nutritional concepts assigned by health professionals and students. J Med Educ. 1979;54(8):607-12.

21. Kris-Etherton PM, Akabas SR, Bales CW, et al. The need to advance nutrition education in the training of health care professionals and recommended research to evaluate implementation and effectiveness. Am J Clin Nutr. 2014;99(Suppl 5):66S.

22. Kris-Etherton PM, Akabas SR, Douglas P, et al. Nutrition competencies in health professionals' education and training: A new paradigm. Adv Nutr. 2015;6(1):83-7.
23. Guagnano MT, Merlitti D, Pace-Palitti V, Manigrasso MR, Sensi S. Clinical nutrition: Inadequate teaching in medical schools. Nutr Metab Cardiovasc Dis. 2001;11(2):104-7.

24. Adams KM, Kohlmeier M, Zeisel SH. Nutrition education in U.S. medical schools: Latest update of a national survey. Acad Med. 2010;85(9):1537-42.

25. Adams KM, Lindell KC, Kohlmeier M, Zeisel SH. Status of nutrition education in medical schools. Am J Clin Nutr. 2006;83(4):4S.

26. Chung M, van Buul VJ, Wilms E, Nellessen N, Brouns, F J P $\mathrm{H}$. Nutrition education in european medical schools: Results of an international survey. Eur J Clin Nutr. 2014;68(7):844-6.

27. Danek RL, Berlin KL, Waite GN, Geib RW. Perceptions of nutrition education in the current medical school curriculum. Family medicine. 2017;49(10):803-6.

28. Oberhelman SS, Cozine EW, Umaretiya PJ, Maxson JA, Thacher TD. Vitamin D and the breastfeeding infant: Family medicine clinicians' knowledge, attitudes, and practices. J Hum Lact. 2018;34(2):331-6.

29. Mogre V, Stevens FC, Aryee PA, Amalba A, Scherpbier AJ. Why nutrition education is inadequate in the medical curriculum? a qualitative study of students' perspectives on barriers and strategies. BMC Med Educ. 2018;18:26.

30. Yu Y, Yang Y, Li Z, et al. The association between medical students' lifestyles and their attitudes towards preventive counseling in different countries. BMC public health. 2015;15:1124.

31. Clarke CA, Hauser ME. Lifestyle medicine: A primary care perspective. J Grad Med Educ. 2016;8(5):665-7.

32. Ball L, Barnes K, Laur C, Crowley J, Ray S. Setting priorities for research in medical nutrition education: An international approach. BMJ open. 2016;6: e013241. 